\title{
Consonant effects on tonal registers in Jiashan Wu
}

\author{
Bing'er Jiang, Jianjing Kuang*
}

\begin{abstract}
Breathy phonation is known as the primary cue of the "voiced" stops in $\mathrm{Wu}$ dialects, and is associated with the lower tonal register. This study discusses the phonetic realization of the tonal register of $\mathrm{Wu}$ dialects by measuring relative prominence of the first harmonic to some higher-frequency components in the spectrum, F0 and periodicity (CPP) of Jiashan Wu monosyllabic words. We find that in Jiashan $\mathrm{Wu}$, the phonetic targets for tonal register contrasts are a steeper spectralslope and a lower F0, which is consistent cross all consonant manners, while the articulatory realization varies among different types of consonants.
\end{abstract}

Keywords. Wu dialect; breathiness; tonal register; consonant effects

1. Introduction. The $\mathrm{Wu}$ dialect is a Chinese dialect spoken in Shanghai, Zhejiang province and southern Jiangsu province of China. Jiashan $\mathrm{Wu}$, along with other $\mathrm{Wu}$ dialects, is a tone language with two registers. This study investigates consonant effects on register contrast in Jiashan Wu. According to Yip $(1980,2002)$, a register feature [+/- Upper] divides the tonal space according to pitch: the register feature [+Upper] indicates a higher pitch range and [-Upper] indicates a lower pitch range. In other words, the upper register always has a higher pitch than the lower one. Yip further proposes that a tone feature [+/-high] subdivides registers into two halves, creating four tones. The two sets of features are able to represent the tonal inventory of the Jiashan dialect. The numbers in parenthesis are Chao numbers, another way of indicating the tonal contour. 5 stands for the highest pitch and 1 stands for the lowest pitch.

\begin{tabular}{ccccc} 
& Ping & Shang & $\mathrm{Qu}$ & $\mathrm{Ru}($ entry) \\
\hline+ Upper & $\mathrm{hl}(53)$ & $\mathrm{h}(44)$ & $\mathrm{hh}(35)$ & $\underline{\mathrm{h}(5)}$ \\
\hline -Upper & $\mathrm{hl}(31)$ & \multicolumn{2}{c}{$\mathrm{h}(13)$} & $\underline{1}(2)$ \\
\hline \hline
\end{tabular}

Table 1: Tone inventory of Jiashan Wu

As shown in Table 1, there are seven tones within two registers in the Jiashan dialect. Ping tone is a falling tone, Shang tone is a high level tone, $Q u$ tone is a rising tone, and $R u$ (also called entry tone) tone is short in vowel duration compared with other tones, and ends in a glottal stop. In the lower register, the Shang and $Q u$ tones have merged and are realized as a rising tone (Yu 1988).

Apart from pitch contrast, it is traditionally believed that there is also a voicing contrast between registers for some syllables, specifically, those with stop and fricative onsets. According to earlier studies (Edkins 1953, Karlgren 1940, Shih 1983) and traditional transcription systems, stops and fricatives in the lower register are voiced, while those in the upper register are voiceless (for stops, either voiceless aspirated or voiceless unaspirated). Table 2 shows the consonant inventory of the Jiashan dialect based on traditional transcriptions with minor corrections in order to adapt them to the IPA.

\footnotetext{
* This study is supported by the URF Award of UPenn to Jianjing Kuang. Many thanks to professor Morgan Sonderegger and Dr. Michael McAuliffe at the linguistics department of McGill for their help on statistical analysis. All errors are solely the authors'. Authors: Bing'er Jiang, McGill University (binger.jiang@mail.mcgill.ca) \& Jianjing Kuang, University of Pennsylvania (kuangj@ling.upenn.edu).
} 


\begin{tabular}{|c|c|c|c|c|c|c|}
\hline & Bilabial & $\begin{array}{l}\text { Labio- } \\
\text { dental }\end{array}$ & Alveolar & $\begin{array}{c}\text { Alveolo- } \\
\text { palatal }\end{array}$ & Velar & Glottal \\
\hline Plosive & $\mathrm{p} \mathrm{p}^{\mathrm{h}} \mathrm{b}$ & & $\mathrm{t} \mathrm{t}^{\mathrm{h}} \mathrm{d}$ & & $\mathrm{k} \mathrm{k}^{\mathrm{h}} \mathrm{g}$ & $?$ \\
\hline Nasal & $\mathrm{m}$ & & $\mathrm{n}$ & $\eta_{6}$ & y & \\
\hline Fricative & & $f_{v}$ & S Z & 6 & $X$ & \\
\hline Lateral & & & 1 & & & \\
\hline Affricate & & & $\mathrm{ts} \mathrm{ts}^{\mathrm{h}}$ & to $\mathrm{tg}^{\mathrm{h}} \mathrm{dt}$ & & \\
\hline
\end{tabular}

(Confer Yu (1988) and Chao (1928) on Jiaxing dialect)

Table 2. Consonant inventory of Jiashan $\mathrm{Wu}$

Voiced obstruents (/b, d, g, z, v, dz/) occur only in the lower register, while voiceless obstruents occur only in the upper register (except for the glottal stop / / which appears only in coda position). Nasals, laterals and / $/$ / can occur in both registers. Previous studies specifically note that stops in $\mathrm{Wu}$ dialect form a three-way contrast: voiced, voiceless unaspirated and voiceless aspirated (Edkins 1853, Karlgren 1940, Shih 1983).

Later studies on initial stops found, however, that there is only a contrast in aspiration, but not in voicing. In particular, voiced stops in the lower register are actually voiceless without vocal fold vibration during closure (Chao 1928, 1934,1935). Moreover, in syllables belonging to the lower register, the stop onset is followed by a vowel with breathy phonation (Cao and Maddieson 1992; Ladedoged and Maddieson 1996; Ren 1988; Zhu 2010). Its counterpart in the upper register, on the other hand, is realized with modal phonation.

Similar to stops, fricatives also turn out to be voiceless in the lower register in phraseinitial position (Chen 2015; Chen and Gussenhoven 2015). In other words, lower-register fricatives are voiceless if produced in isolation, and are voiced only when preceded by another syllable.

The breathy-modal distinction between the upper and lower registers holds true for other onsets as well. Sonorants and zero-onsets (i.e. onsetless syllables, for example /a 31/ 'shoe') are reported to carry a breathy vowel in the lower register (Sherard 1972; Qian 1992; Zhu 2005), and later studies (Zhu 2005; Zhang \& Yan 2015) validate breathy phonation in in syllables with fricative onsets as well.

In short, the two registers in $\mathrm{Wu}$ dialect contrast in both pitch and phonation: the upper register is realized with high pitch and modal phonation, while the lower register has a lower pitch range and breathy phonation. The voicing contrast turns out to be non-existent for phraseinitial syllables with stop or fricative onsets.

The current study examines consonant effects on register contrast in Jiashan Wu. While studies show that register contrast occurs with all types of onsets, it is not clear whether or not the contrast is acoustically realized the same way for different onset manners. It is possible that syllables with different onsets have different acoustic properties in the register contrast. In the following parts of this paper, section 2 gives theoretical predictions about possible consonant effects and summarizes relevant past studies; section 3 discusses acoustic measures indicating breathy phonation cross-linguistically; section 4 describes the methods adopted in this study; section 5 presents the results and section 6 discusses the findings. 
2. Consonant effects on register contrast. First, stop and fricative onsets are possible to pattern differently from sonorants (i.e. liquids and nasals) and zero-onsets. Among all five types of onsets, phrase-initial stops and fricatives are voiceless in the lower register, while sonorants are voiced. Moreover, the laryngeal configuration is different in producing fricatives, stops and sonorants. It is possible that obstruents (i.e. fricatives and stops) pattern differently from sonorants because of their voicelessness, and fricatvies might also behave differently from stops because of the continuous noise in the production.

Second, it is possible that the two sonorant onsets (i.e. liquids and nasals) behave differently. In particular, nasalization is similar to breathiness acoustically. Nasals have decreased lowfrequency prominence and an enhanced first harmonic. These particular properties are very similar to the acoustics of breathiness, as indicated by specific measures of the spectral components (more details below). It is also suggested that nasalization and spread-glottis enhance each other (Keyser and Stevens 2006). Studies (e.g., Klatt \& Klatt 1990) do find that speakers were sometimes unable to distinguish breathiness from nasalization. Thus, syllables with nasal onsets might be more breathy than other sonorants.

Third, zero-onset syllables might follow a pattern different from the two sonorants. While breathiness is a common property of the lower register for all three non-obstruent-initial syllables, it is reported that zero onsets are occasionally preceded by glottalization in upper register words (Qian 1992; Yip 1993; Zhu 2005), which may lead to a larger register contrast. In other words, zero-onset syllables are not modal in either register: the upper register is glottalized (at least at the beginning of the production) rather than modal and the lower register is breathy. This is likely to be reflected by various acoustic measures, which are discussed in detail in the following section.

Studies on various varieties of $\mathrm{Wu}$ dialects also found differences among different manners of onset consonants. Rose (1989) finds that in Zhenhai Wu, "whisper" is conditioned by syllableinitial obstruent, and whispery voicing (distinct from whisper) appears only on syllable initial sonorants. Shanghainese, a subdialect of Wu previously known to have a phonatory contrast, is reported to show phonation correlates only in fricatives (Zhang \& Yan 2015). There are also dialectal differences: in the Yongjiang dialect, fricatives, stops and zero-onsets can be "growl", and in the Jinyun dialect, words with stop and nasal onsets pattern together in breathiness (Rose 2015).

3. Acoustic correlates of breathy voice. Breathiness is the result of incomplete glottal closure during the 'closed' phase of the phonatory cycle (Ladefoged 1971; Ladefoged \& Maddieson 1996), its specific round near-sinusoidal shape waveforms leads to a relatively high amplitude of the first harmonic (H1) and relatively weak upper harmonics (Hillenbrand et al. 1994, citing Bickley 1982; Huffman 1987; Klatt \& Klatt 1900).

Various acoustic and perceptual studies have examined the $\mathrm{H} 1$ enhancement in breathy production, and while some found such effects, others did not. Ladefoged (1983) finds H1 enhancement in !Xóõ's breathy words, and a later perception study (Ladefoged \& AmtonanzasBarroso 1985) further proved the effect. Huffman (1987) also finds increased H1 in Hmong breathy samples. However, there are also studies that suggest $\mathrm{H} 1$ alone might not be enough as an indicator of breathiness. By using synthetic Gujarati words in the perception experiment, Bickley (1982) shows that while speakers were able to distinguish breathy/clear word pairs by the $\mathrm{H} 1$ difference, the needed $\mathrm{H} 1$ distinction is greater than the natural difference. Despite her 
belief that H1 was the most robust cue for breathiness, Fischer-Jorgensen (1967) concluded that $\mathrm{H} 1$ alone is not sufficient for distinguishing breathiness.

Studies above show that while H1 is a potential indicator of breathy production, it is not convincing to use it as the sole indicator of breathiness cross-linguistically (Klatt \& Klatt 1990; Fischer-Jorgensen 1967); moreover, languages differ in specific acoustic parameters in reflecting breathiness, and it is proposed that different languages need different (sets of) acoustic measures in distinguishing breathy-modal contrast (Keating \& Esposito 2006; Wayland \& Jongman 2003; Chen 2010).

Among various acoustic parameters, $\mathrm{H} 1-\mathrm{H} 2$, the difference of amplitude between the first and second harmonics, is a strong indicator of breathiness. From the point of view of articulation, breathy production always results from vocal fold vibration during incomplete closure. Such articulatory characteristics can be captured by a relatively high value of open quotient (OQ), the proportion of the glottal period where the vocal folds are not in contact. Since H1-H2 is correlated with OQ (Esposito 2006, 2010; Kreiman, Gerratt \&Antoñanzas-Barroso 2007; Wayland \& Jongman 2003; Holmberg et al 1995), it is able to distinguish breathy phonation.

Along with Klatt \& Klatt (1990)'s suggestion that H1 alone is not appropriate to distinguish breathy phonation and should be compared with other spectral components, studies found that the relative amplitude between $\mathrm{H} 1$ and the first three formants (i.e. H1-A1, H1-A2, H1-A3) are successful in denoting breathiness with different importance in different languages. H1-A1, a reflection of posterior glottal opening (Hanson et al. 2001) is found to distinguish the upper and lower register in Wu dialect (Cao and Maddieson 1992); H1-A2 is found to better distinguish breathy from modal production in Chong (Blankenship 2002) and Tsonga (Trail \& Jackson 1987), while H1-A3 is more successful in Takhian Thong Chong (DiCanio 2009).

In addition, Cepstral peak prominence (CPP), a measure of periodicity, is also widely used in evaluating phonation distinction (Blankenship 2002; Esposito 2006, 2010), and is found to be a reliable cue cross-linguistically (Esposito 2006, 2010). Hillenbrand et al. (1994) investigated English speakers producing modal, moderately breathy, and very breathy phonation and found that CPP is the most accurate prediction of perceived breathiness.

In terms of $\mathrm{Wu}$ dialects, there is no agreement on which specific acoustic parameters best cue breathiness. While some studies observe a significant difference between two registers in H1-H2 (Cao \& Maddieson 1992; Ladefoged \& Maddieson 1996, Hu 2001), others do not find it to be a reliable cue (Zhu 2007, Chen 2010). Apart from H1-H2, other parameters are also reported to be able to distinguish registers: H1-A1 is found significant by Cao \& Maddieson (1992), and so is $\mathrm{H} 1-\mathrm{A} 3$ in distinguishing the register contrast including Wenzhou $\mathrm{Wu}(\mathrm{Hu} 2001)$ and Shanghai Wu (Chen 2010).

This study examines the register contrast in the Jiashan Wu dialect with a focus on consonant effect. Specifically, it investigates whether or not the Jiashan dialect has phonation contrast between the two tonal registers, and if yes, which measures distinguish the contrast. More importantly, this study explores whether or not the register contrast is realized the same way among the five types of onsets.

4. Methods. The wordlist consisted of 60 falling tone monosyllabic words with all possible combinations of the five consonant categories as onsets (i.e. /f, v, s, z/ for fricatives, /1/ for liquid, $/ \mathrm{m} /$ and $/ \mathrm{n} /$ for nasals, $/ \mathrm{p}, \mathrm{p}^{\mathrm{h}}, \mathrm{t}, \mathrm{t}^{\mathrm{h}}, \mathrm{k}, \mathrm{k}^{\mathrm{h}} /$ for stops, and zero); four vowels as the nucleus (i.e. /a, $\varepsilon$, 
i, $\mathrm{o} /$ ); and two registers (i.e. the upper and lower). The wordlist was designed to strike a balance so that there would be multiple syllables with various kinds of onsets and nuclei in both registers. Production data were elicited from 12 speakers (six females and six males), aged 32-55. Participants produced each word three times in isolation.

After collecting the data, the beginning and end position of each vowel were annotated. Acoustic measures were made automatically using VoiceSauce (Shue et al. 2011): F0, H1 and relative prominence of $\mathrm{H} 1$ (i.e. $\mathrm{H} 1{ }^{*}-\mathrm{H} 1 *, \mathrm{H} 1{ }^{*}-\mathrm{A} 1 *$, $\mathrm{H} 1{ }^{*}$-A2*, $\mathrm{H} 1{ }^{*}-\mathrm{A} 3 *$ ) and $\mathrm{CPP}$ (cepstral prominence peak). All these parameters were measured at nine evenly divided time points from the beginning to the end of the vowel, and the analysis is based on the mean value of the nine measurements.

5. Results. This section presents the seven models fitted for the relevant acoustic measures and reports the model predictions respectively.

5.1. F0. Jiashan $\mathrm{Wu}$ is a tone language with two pitch registers, and is expected to show a contrast in F0. Statistical analyses are based on mixed-effects modeling (Baayen et al. 2008) using the lmer function in the lme4 package (Bates et al. 2011). For the current model, the response variable is Normalized F0, and the fixed independent variables are consonant Categories, Register, Vowel, Gender, and the interaction between consonant and register, and all levels of the independent variables used the groupings described in the previous section. The by-speaker random slopes include consonant Category, Register, the interaction between the two, and Vowel. The by-item random slope is Gender. Fixed variables were sum coded and centered, and hence the intercept represents the grand mean.

The model shows that there are significant effects for Register $(\beta=0.282867, \mathrm{SE}=0.055643$, $\mathrm{p}<0.001)$ and Gender $(\beta=-1.758275, \mathrm{SE}=0.243742, \mathrm{p}<0.001)$. In other words, the F0 of the lower register is consistently lower than that of the upper register, and males have lower a F0 compared to females across all onset types. As the upper register phonologically stands for a higher pitch range and the lower register a lower pitch range, the results are expected. Similarly, the considerable lower F0 values of males are in correspondence with the physical nature of the vocal tracts. The described findings are observable in Figure 1.

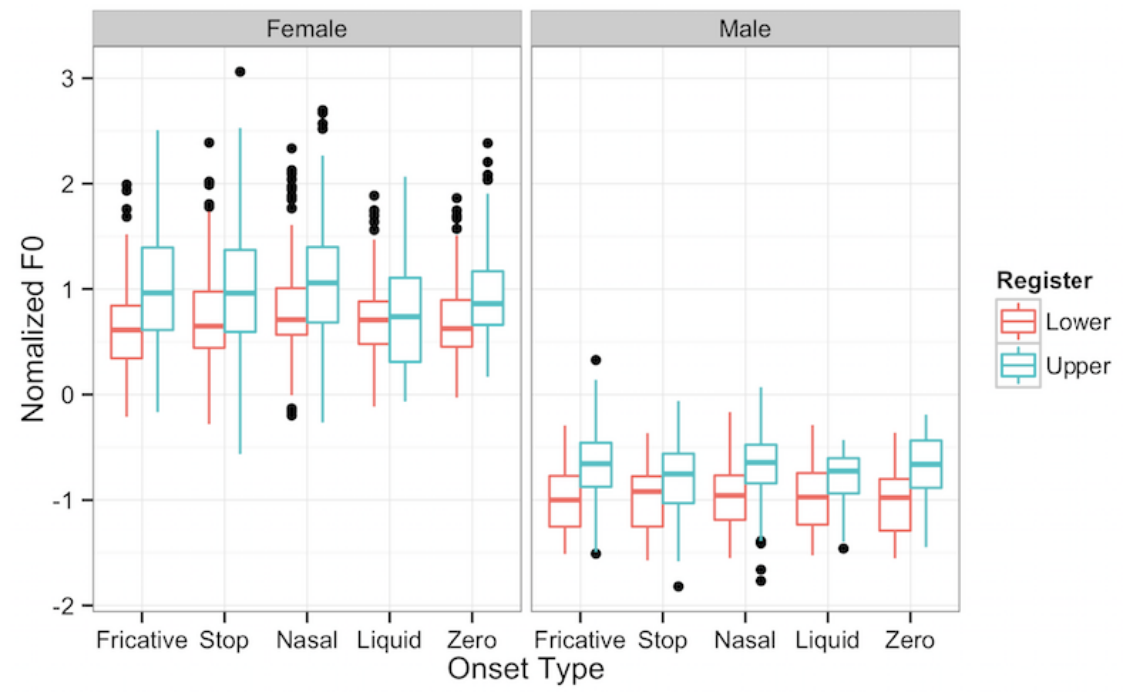

Figure 1. Summary of F0 across onset types. The left panel shows results of females' production and the right panel shows males'. Red and green boxes represent the lower and upper register 
respectively. Groupings in the x-axis are made according to onset types: fricatives, liquids, nasals, stops and zero-onsets. The y-axis stands for corresponding mean F0 values of syllables with the specific onsets.

With regard to consonant types and the interaction between consonants and registers, neither of the two shows a significant effect. In other words, no consonant types have considerably higher or lower pitch compared to other onsets, and nor do they differ significantly between the two registers.

5.2. $\mathrm{H} 1 *$. The model fitted for $\mathrm{H} 1 *$ is the same as the F0 model, except for replacing the response variable $\mathrm{F} 0$ with $\mathrm{H} 1 *$. A higher $\mathrm{H} 1$ * value potentially indicate a breathier production.

The model shows that there are significant effects for Register $(\beta=-0.646361, \mathrm{SE}=$ $0.086757, \mathrm{p}<0.001)$ and Gender $(\beta=1.365871, \mathrm{SE}=0.228522, \mathrm{p}<0.001)$. In other words, the breathy lower register always has higher $\mathrm{H} 1$ value than the modal upper register, and the value for males is higher than females. The above findings are shown in Figure 2.

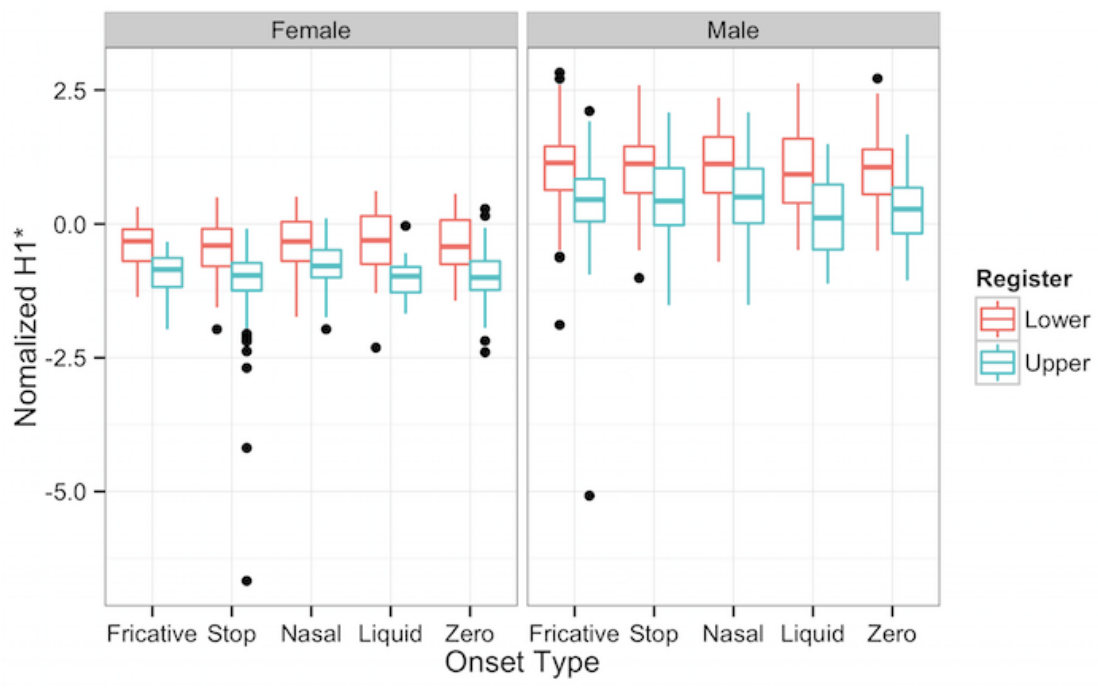

Figure 2. Summary of $\mathrm{H} 1 *$ across onset types

Among onset types, the level representing the difference between Nasal and the intercept (grand mean) is significant $(\beta=0.102473, \mathrm{SE}=0.042481, \mathrm{p}=0.02144)$. Moreover, Nasal is the only level (i.e. onset type) that has a positive coefficient (i.e. higher than the grand mean). This suggests that nasals are significantly different from the mean value of all onsets, and have the highest $\mathrm{H} 1$ value compared with the rest of the onsets.

As for consonant-tone interaction, none of the levels reach significance, indicating that there is no significant $\mathrm{H} 1 *$ difference in register contrast among the five onset types.

5.3. $\mathrm{H} 1 *-\mathrm{H} 2 *$. The model fitted for $\mathrm{H}_{1} *-\mathrm{H} 2 *$ measure is the same with the previous models, except for replacing the response variable with $\mathrm{H} 1{ }^{*}-\mathrm{H} 2 *$. Given the correlation between $\mathrm{H} 1 *_{-}$ $\mathrm{H} 2 *$ and $\mathrm{OQ}$, the higher the value, the breathier the production. According to the model prediction, none of the predictors is significant except for the level indicating the difference between Nasal and the grand mean. 
As reflected in Figure 3, the values of two registers largely overlap, and there is not much difference between males and females. This is in correspondence with the model prediction that neither Gender nor Register is significant.

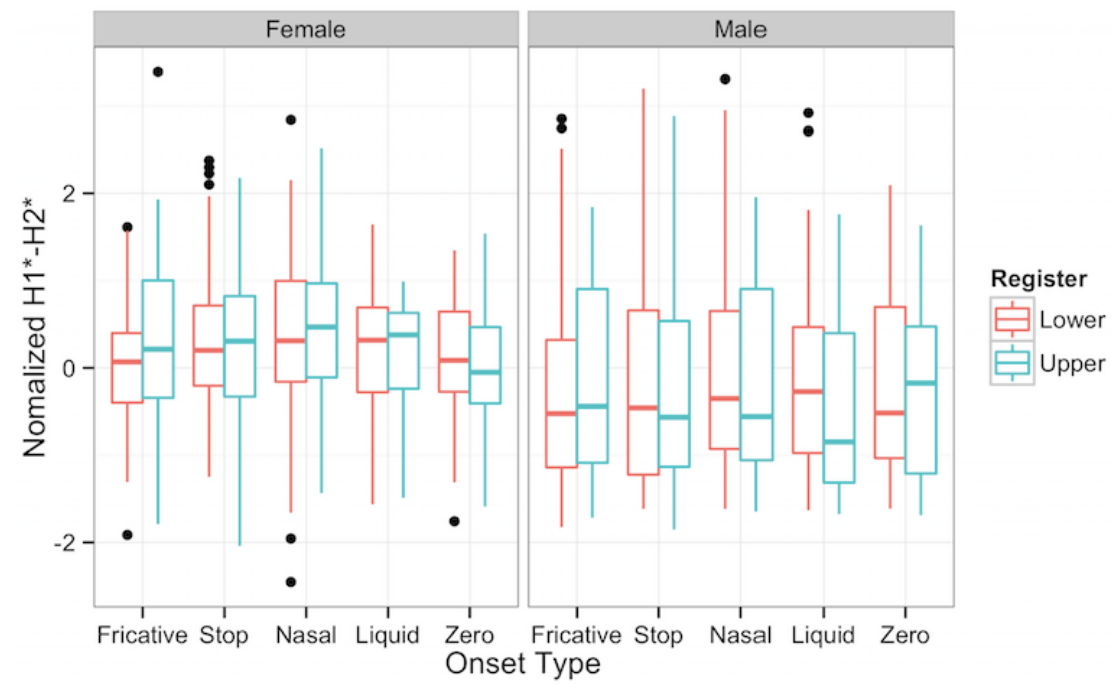

Figure 3. Summary of $\mathrm{H} 1{ }^{*}-\mathrm{H} 2 *$ across onset types

Concerning the effect of onset types, Nasal is again significant $(\beta=0.113506, \mathrm{SE}=$ $0.049218, p=0.0364$ ), and shows the largest positive coefficient compared with other levels. This implies that, similar to the $\mathrm{H} 1 *$ model, Nasals are significantly different from the grand mean, and have the highest value across all types of onsets.

5.4. $\mathrm{H} 1 *-\mathrm{A} 1 *$. The model fitted for $\mathrm{H} 1 *-\mathrm{A} 1 *$ measure is the same with the previous models, except for replacing the response variable with $\mathrm{H} 1 *-\mathrm{A} 1 *$, a measure for spectral tilt. A larger number indicates a breathier voice.

The model predicts that there is no significant effect for Gender, and a mild effect for Register $(\beta=-0.25423, \mathrm{SE}=-0.25423, \mathrm{p}=0.05212)$. Figure 4 indeed reflects the predictions by the model: there is not much difference between the two registers, nor between genders.

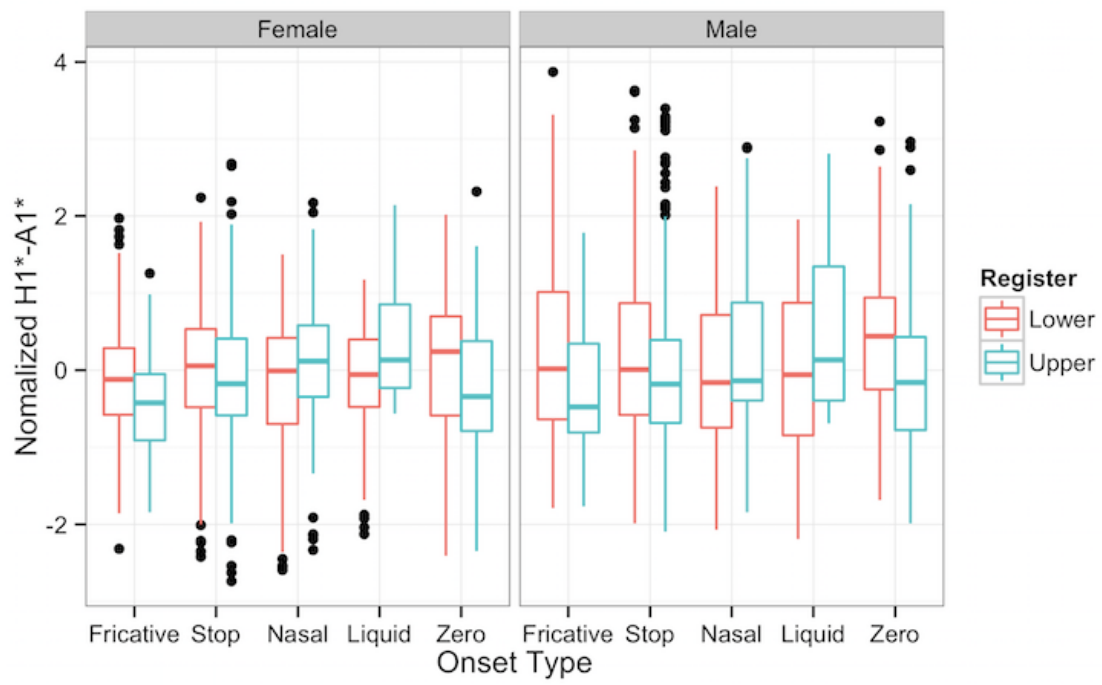

Figure 4 . Summary of fixed effects of $\mathrm{H} 1 *-\mathrm{A} 1 *$ across onset types 
While there is no significance for consonant-register interactions, the Nasal category is again significant $(\beta=0.17008, \mathrm{SE}=0.17008, \mathrm{p}=0.00497)$. Moreover, it has again the highest value among other onset types. As for consonant-register interaction, none is significant, an implication of no consonant effects for $\mathrm{H} 1 *-\mathrm{A} 1 *$ values in register contrast.

5.5. $\mathrm{H} 1 *-\mathrm{A} 2 *$. The model fitted for $\mathrm{H} 1 *-\mathrm{A} 2 *$ measure is the same with the previous models, except for replacing the response variable with $\mathrm{H} 1{ }^{*}-\mathrm{A} 2 *$, another measure for spectral tilt, where a higher value implies breathier phonation.

There is a significant effect of Register $(\beta=-0.304712, \mathrm{SE}=0.096963, \mathrm{p}=0.00729)$, but not of Gender. This is shown in Figure 5, the lower registers tend to have higher values in general, though not without exception, while results for males and females largely overlap.

Moreover, there is a mild effect of the Nasal level in Category $(\beta=0.085618, \mathrm{SE}=0.048981$, $\mathrm{p}=0.09141$ ). In a similar vein, given its highest positive coefficient, the $\mathrm{H} 1 *-\mathrm{A} 2 *$ value for Nasal is the highest among all types of onsets.

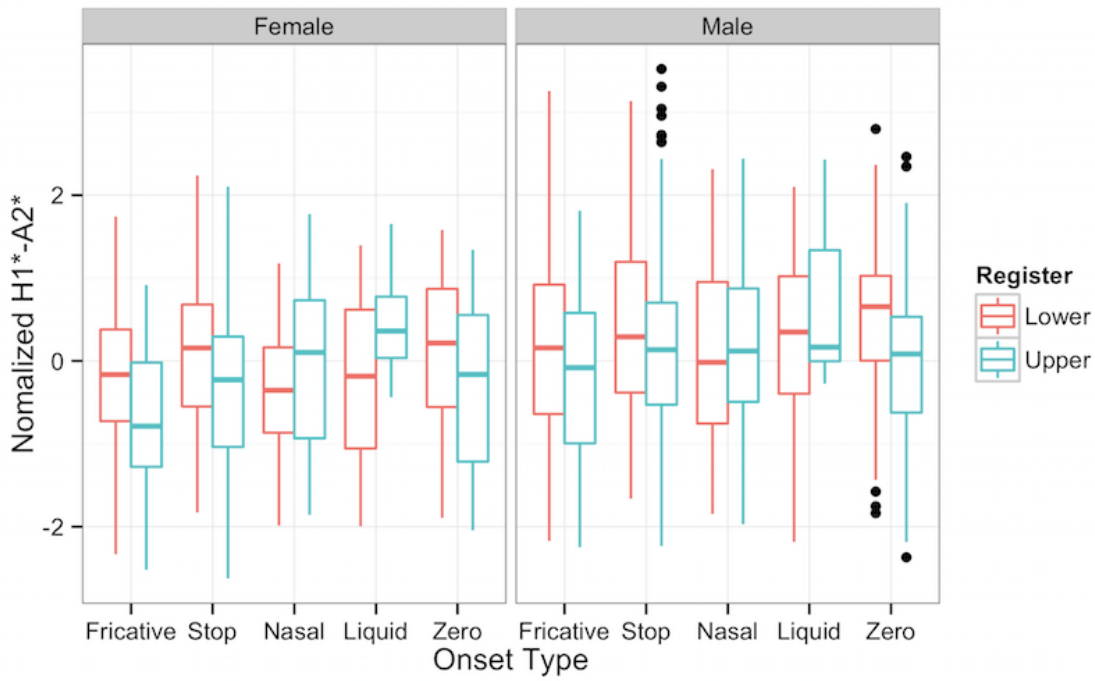

Figure 5. Summary of $\mathrm{H} 1 *-\mathrm{A} 2 *$ across onset types

5.6. $\mathrm{H} 1 *-\mathrm{A} 3 *$. The model fitted for $\mathrm{H} 1 *-\mathrm{A} 3 *$ measure is the same with the previous models, except for replacing the response variable with $\mathrm{H} 1{ }^{*}-\mathrm{A} 3 *$. $\mathrm{H} 1{ }^{*}$ - $\mathrm{A} 3 *$ is also a measure of spectral tilt, and the higher the value (a steeper tilt), the breathier the production.

The only significant predictor is Register $(\beta=-0.275737, \mathrm{SE}=0.122812, \mathrm{p}=0.040503)$. As observed from Figure 6, the lower register always has higher value than the upper register. There is not much difference between genders, and the model does not predict Gender as significant either. 


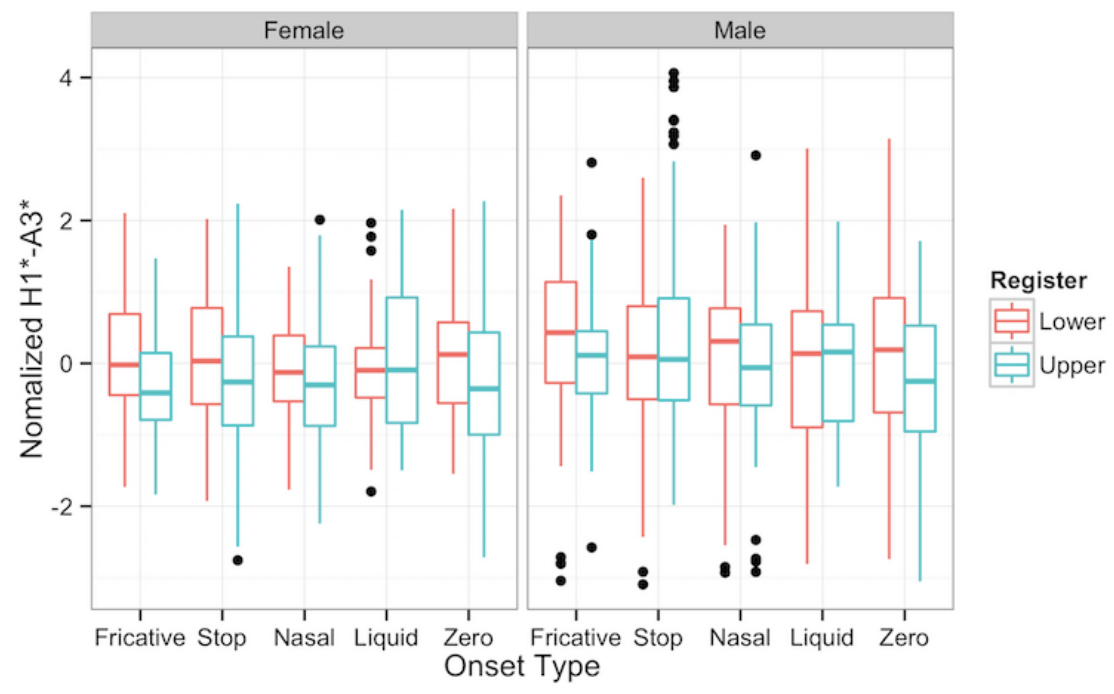

Figure 6. Summary of $\mathrm{H} 1 *-\mathrm{A} 3 *$ across onset types

5.7. CPP. The model fitted for CPP measure is the same with the previous models, except for replacing the response variable with CPP. A high CPP means high periodicity, representing modal phonation, while a low CPP refers to non-modal phonation, no matter if it is breathy or creaky.

There are no significant effects in terms of Category (i.e. onset types) or Gender. However, significant effects are found for Register $(\beta=-0.324412, \mathrm{SE}=0.104251, \mathrm{p}=0.00622)$ and the consonant-register interaction. Specifically, the upper register has lower CPP value, as observable in Figure 7. As for the consonant-register interaction, the Fricative*Register level is significant ( $\beta=$ $-0.281550, \mathrm{SE}=0.125720, \mathrm{p}=0.03076)$, and the $\mathrm{Stop} *$ Register level has a mild effect $(\beta=-$ $0.173594, \mathrm{SE}=0.093583, \mathrm{p}=0.07096$ ). It is important to note that, while the coefficients of Stops and Fricatives are negative, suggesting smaller register contrasts, the coefficients of sonorants and Zero-onset are positive, suggesting larger register contrasts. Moreover, the non-significance of the sonorant levels reveals that Nasals and Liquids pattern with Zero-onsets.

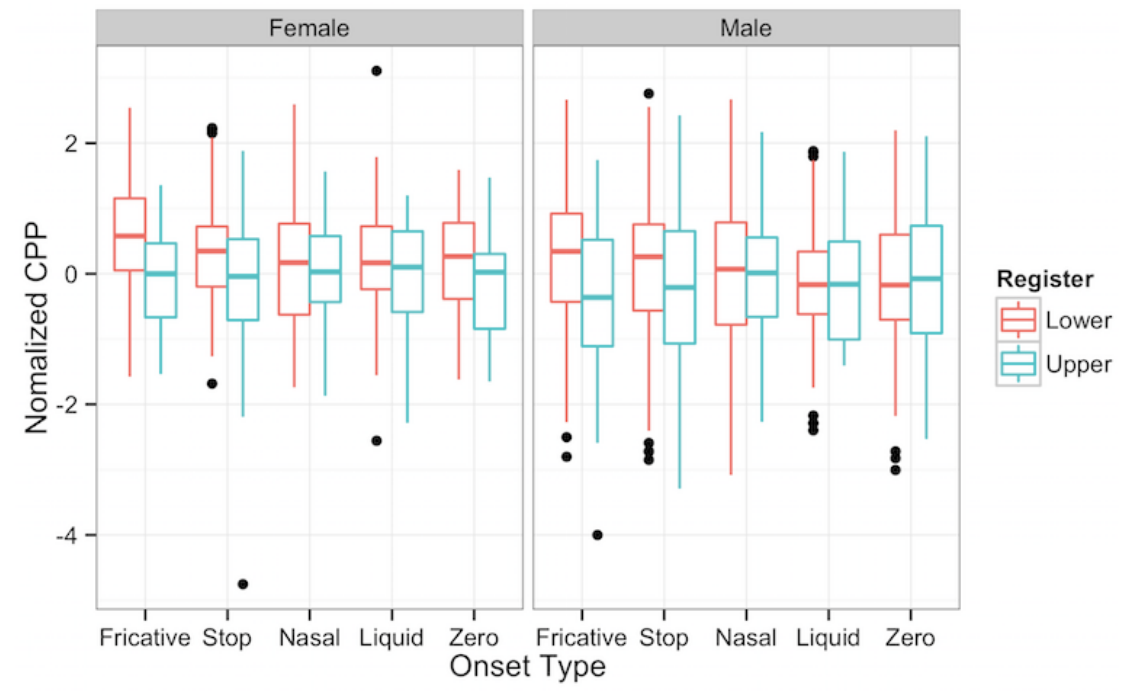

Figure 7. Summary of CPP across onset types 
To summarize, all measures except $\mathrm{H} 1 *_{-} \mathrm{H} 2 *$ (and $\mathrm{H} 1{ }^{*}-\mathrm{A} 1 *$, to a lesser extent) successfully distinguishes two registers. Only $\mathrm{H} 1 *$ and $\mathrm{F} 0$ are able to predict gender differences. Moreover, Nasals show significant effect for low frequency measures (i.e. $\mathrm{H} 1 *$, $\mathrm{H} 1{ }^{*}-\mathrm{H} 2 *, \mathrm{H} 1{ }^{*}$-A 1 *, and a mild effect in $\left.\mathrm{H} 1{ }^{*}-\mathrm{A} 2 *\right)$. The consonant-register interaction is only observed for Fricatives and Stops in CPP. Specifically, obstruents have smaller phonation contrast between registers, and sonorants and zero-onsets pattern together.

6. Discussion. According the results, Wu dialect has redundant pitch and phonation contrast between the upper and lower register: the upper register has higher pitch and is modal, while the lower register has lower pitch and is breathy.

F0 results show that there is a pitch contrast between registers, which supports the register feature proposed by Yip. However, the results do not show significant difference among different onset types, nor their interaction with registers, which is to say that the pitch contrast between the two tonal registers are realized in the same way among the five onset types.

In terms of breathiness measures, all acoustic parameters distinguish the two registers except for $\mathrm{H} 1{ }^{*}-\mathrm{H} 2 *$ (and $\mathrm{H} 1{ }^{*}-\mathrm{A} 1 *$, to a lesser extent), suggesting that $\mathrm{H} 1{ }^{*}-\mathrm{H} 2 *$ is not a reliable cue for breathiness of in $\mathrm{Wu}$ dialect. In fact, while it is said to be a robust cue for breathiness in many languages, it is not uncommon that $\mathrm{H} 1 *-\mathrm{H} 2 *$ fails to distinguish breathy voice from modal voice. For example, it is not successful in predicting breathy phonation in Chong, Mon and Tamang (Blankenship 2002; Esposito 2006). Since H1*-H2* is correlated with OQ, the failure of $\mathrm{H} 1 *-\mathrm{H} 2 *$ distinction suggests that Jiashan Wu might be among those languages that do not show a discernible open phase in breathy production compared with the modal one. The non-universal predictability of $\mathrm{H} 1{ }^{*}-\mathrm{H} 2 *$ further implies that those so-called "breathy voice" might not necessarily be characterized by the same feature (i.e. longer open phase); at least not for the Jiashan Wu dialect, where there is no significant difference in open phase between modal and breathy phonation.

Spectral information of low and lower-to-mid range measures $\left(\mathrm{H} 1 *, \mathrm{H} 1{ }^{*}-\mathrm{H} 2 *, \mathrm{H} 1 *-\mathrm{A} 1 *\right.$, and a mild effect in $\mathrm{H} 1 *-\mathrm{A} 2 *$ ) show significance in Nasals. A look at the summaries of the particular models reveals that nasals have the highest coefficients, indicating that they induce the most breathy production on the following vowel. However, there is no significant interaction between Nasal and Register, demonstrating that while vowels following nasal onsets are generally more breathy in both registers, they have a similar phonation contrast as other sonorant onsets. One possible explanation could be drawn from the enhancement theory (Keyser \& Stevens 2006), which proposes that the enhancement process can add extra motoric instructions to make the jeopardized features more salient, and in the current case, nasalization and breathiness.

Mid and high range measures of spectral tilt, that is, $\mathrm{H} 1 *-\mathrm{A} 2 *$ and $\mathrm{H} 1 *-\mathrm{A} 3 *$, also show a significant register difference. However, there is no difference among onset types (except for the very small effect of $\mathrm{H} 1 *-\mathrm{A} 2 *)$, or in their interaction with registers. This again reveals that all onset types behave similarly in terms of the velocity at which vocal folds return from the open status. Moreover, the non-significant effect of Gender in the $\mathrm{H} 1{ }^{*}-\mathrm{A} 2 *$ and $\mathrm{H} 1 *-\mathrm{A} 3 *$ model suggests that males and females have similar vocal fold closing velocity. In addition, spectral tilt is an indication of the time the vocal folds take to return from the open to closed status: the longer the time, the steeper the spectral tilt. The articulatory nature of breathy voice in $\mathrm{Wu}$ dialect, 
along with other languages that contrast in spectral tilt measures, is a relatively slower velocity for vocal folds to return to the closed status.

The other acoustic measure, CPP, also distinguishes the two registers. Moreover, while there is no onset type difference, it shows that fricatives and stops are significantly different in the consonant-register interaction. Specifically, fricatives and stops are the only two onsets that show a negative coefficient in consonant-register interaction. This means that for fricatives and stops, the difference between the lower and upper register is smaller compared with other onset types. Studies find that the upper register for zero-onset syllables (e.g. Qian 1992) begins occasionally with a glottal stop, inducing a tenser production (i.e. a less modal phonation). While this potentially results in a lower CPP and hence a smaller contrast, the CPP distinction between registers for fricatives and stops is even smaller. This suggests that the duration of glottalization is short and quickly changes to modal phonation, which does not affect much of the manner of the whole production. A possible explanation for fricatives and stops to have smaller contrast might be that the two obstruents introduce more noise in the upper register than the sonorants and zeroonset. This leads to a larger noise excitation, which is represented by lower CPP.

7. Conclusion. This study examines the tonal register contrast in Jiashan Wu dialect with a focus on consonant (onset) effects. Jiashan dialect is expected to show both pitch and phonation contrast, which are found from the results of a series of acoustic measures: the F0 results show a pitch contrast between registers, and all measures except for $\mathrm{H} 1 *-\mathrm{H} 2 *$ distinguish breathy from modal phonation. Along with the failure of $\mathrm{H}_{1}{ }_{-} \mathrm{H}_{2} *$ predictability, the nature of breathy voice in $\mathrm{Wu}$ dialect might be a slower closing velocity of the vocal folds (indicated by spectral tilt) and larger presence of noise (indicated by CPP), rather than a longer open phase (indicated by $\mathrm{H}^{*}$ $\mathrm{H} 2 *)$. In terms of consonant effects, nasal-onset syllables are significantly more breathy, possibly because of the acoustic similarity of breathiness and nasalization. As for consonant-register interaction, the only effect is found in CPP measures: fricatives and obstruents have significantly smaller register contrast, while sonorants and zero-onsets pattern together.

This study examines the mean value of the acoustic measures of the whole production.

While breathiness is a common property for the lower tonal register, studies show that the degree of breathiness might change across time (e.g., Cao \& Maddieson 1992; Chen 2010). Future studies should incorporate the time series to better understand the register contrast.

\section{References}

Baayen, R. Harald., Davidson, Douglas J. \& Bates, Douglas M. 2008. Mixed-effects modeling with crossed random effects for subjects and items. Journal of memory and language 59(4), 390-412.

Bates, Douglas, et al. 2007. The lme4 package. $R$ package version 2(1).

Bickley, Corine. 1982. Acoustic analysis and perception of breathy vowels." Speech communication group working papers 1, 71-81.

Blankenship, Barbara. 2002. The timing of nonmodal phonation in vowels. Journal of Phonetics 30(2), 163-191.

Cao, Jianfen., \& Maddieson, Ian. 1992. An exploration of phonation types in Wu dialects of Chinese. UCLA Working Papers in Phonetics, 72, 139-160.

Chao, Yuanren. 1928. Studies in the Modern Wu-dialects. Center for Chinese Research Materials, Association of Research Libraries. 
Chao, Yuanren. 1934 The non-uniqueness of phonemic solutions of phonetic systems. Bulletin of the institute of History and Philology 4:4, 363-390.

Chao, Yuanren. 1935. Types of plosives in Chinese dialects, Bulletin of the Institute of History and Philology, 5:4, 515-520.

Chen, Zhongmin. 2010. An Acoustic Study of Voiceless Onset Followed by Breathiness of Wu Dialects: Based on the Shanghai Dialect. Studies in Language and Linguistics, 30:3, 20-34.

Chen, Zhongmin. 2015. Breathy voice and low tone. Journal of Chinese Linguistics 43(1), 90117. Chinese University Press.

Chen, Yiya., \& Gussenhoven, Carlos. 2015. Shanghai Chinese. Journal of the International Phonetic Association, 45(03), 321-337. http://dx.doi.org/10.1017/S0025100315000043.

DiCanio, Christian T. 2009. The phonetics of register in Takhian Thong Chong. Journal of the International Phonetic Association 39(02), 162188. http://dx.doi.org/10.1017/S0025100309003879.

Edkines, Joseph. 1868. A Grammar of Colloquial Chinese, as Exhibited in the Shanghai Dialect. $2^{\text {nd }}$ edition. Shanghai: Shanghai Presbyterian Mission Press.

Esposito, Christina. 2006. The effects of linguistic experience on the perception of phonation. Ph.D. thesis, UCLA.

Esposito, Christina M. 2010. The effects of linguistic experience on the perception of phonation. Journal of Phonetics 38(2), 306-316.

Fischer-JPrgensen, Eli. 1967. Phonetic analysis of breathy (murmured) vowels in Gujarati. Indian Linguistics 28, 71-139.

Hanson, Helen M. et al. 2001. Towards models of phonation. Journal of Phonetics 29(4), 451480.

Hillenbrand, James, Cleveland, Ronald A. \& Erickson, Robert L. 1994. Acoustic correlates of breathy vocal quality. Journal of Speech, Language, and Hearing Research 37(4), 769-778.

Holmberg, Eva B. et al. 1995. Comparisons among aerodynamic, electroglottographic, and acoustic spectral measures of female voice. Journal of Speech, Language, and Hearing Research 38, 1212-1223.

$\mathrm{Hu}$, Fang. 2001. An acoustic analysis of voiced stops in Wenzhou dialect. Proceedings of the 5th national modern phonetic conference.

Huffman, Marie K. 1987. Measures of phonation type in Hmong. The Journal of the Acoustical Society of America 81(2), 495-504.

Karlgren K. Bernhard J. 1940. Zhongguo yinyunxue yanjiu. Trans. Chao, Yuen-ren, Li, Fang kuei and Luo, Changpei. Changsha: The Commercial Press.

Keating, Patricia A., \& Esposito, Christina. 2006. Linguistic voice quality. Proceedings of the Eleventh Australasian International Conference on Speech Science and Technology.

Keyser, Samuel Jay, \& Stevens, Kenneth Noble. 2006. Enhancement and overlap in the speech chain. Language, 33-63.

Klatt, Dennis H., \& Klatt, Laura C. 1990. Analysis, synthesis, and perception of voice quality variations among female and male talkers. the Journal of the Acoustical Society of America $87(2), 820-857$.

Kreiman, Jody., Gerratt, Bruce R. \& Antoñanzas-Barroso, Norma. 2007. Measures of the glottal source spectrum. Journal of Speech, Language, and Hearing Research 50, 595-610.

Ladefoged, Peter. 1971. Preliminaries to linguistic phonetics. Chicago: University of Chicago Press. 
Ladefoged, Peter. 1983. The linguistic use of different phonation types. Vocal fold physiology: Contemporary research and clinical issues, 351-360.

Ladefoged, Peter, \& Antoñanzas-Barroso, Norma. 1985. Computer measures of breathy phonation. UCLA Working Papers in Phonetics 61, 79-86.

Ladefoged, Peter. \& Maddieson, Ian. 1996. The sounds of the world's languages. Cambridge, MA: Blackwell.

Qian, Nairong. 1992. Studies on Contemporary Wu. Shanghai: Shanghai Education Press.

Ren, Nianqi. 1988. A fiberoptic and transillumination study of Shanghai stops. Proceedings of the International Conference on Wu dialects, Hong Kong: 12-14.

Rose, Phil. 1989. Phonetics and phonology of Yang tone; phonation types in Zhenhai. Cahiers de linguistique-Asie orientale 18(2), 229-245.

Rose, Phil. 2015. Tonation in Three Chinese Wu dialects. Proceedings of the 18th International Congress of Phonetic Sciences. Glasgow, UK: the University of Glasgow.

Sherard, Micahel. 1972. Shanghai phonology. Cornell Univ.

Shih, Feng. 1983. The acoustics of voiced stops in Suzhou dialect. Studies in language and linguistics.

Traill, Anthony \& Jackson, Michael. 1987. Speaker variation and phonation types in Tsonga nasals. UCLA Working Papers in Phonetics 67, 1-29.

Wayland, Ratree, \& Jongman, Allard. 2003. Acoustic correlates of breathy and clear vowels: The case of Khmer. Journal of Phonetics 31(2), 181-201. http://dx.doi.org/10.1016/S00954470(02)00086-4.

Yip, Moira. J. 1980. The tonal phonology of Chinese. Doctoral dissertation, Massachusetts Institute of Technology.

Yip, Moira. 1993. Tonal register in East Asian languages. The phonology of tone: The representation of tonal register 245, 268.

Yip, Moira. 2002. Tone. Cambridge University Press.

$\mathrm{Yu}$, Guangzhong. 1988. Syllabary of homophones in Jiaxing dialect. Dialect, 3: 195-208.

Zhang, Jie. \& Yan, Hanbo. 2015. Contextually dependent cue weighting for a laryngeal contrast in Shanghai Wu. Proceedings of the 18th International Congress of Phonetic Sciences. Glasgow, UK: the University of Glasgow.

Zhu, Xiaonong. 2005. An experimental study in Shanghai tones. Shanghai Education Press.

Zhu, Xiaonong. 2007. About Nasal. Studies in Language and Linguistics, 27:3, 1-13.

Zhu, Xiaonong. 2010. Phonetics. Beijing: The Commercial Press. 